\title{
Bone marrow-derived mesenchymal stem cells inhibit the proliferation of hepatic stellate cells by inhibiting the transforming growth factor $\beta$ pathway
}

\author{
LI-TING ZHANG $^{1,2^{*}}$, XUE-QIN FANG ${ }^{3 *}$, QING-FENG CHEN ${ }^{4}$, HONG CHEN $^{1,2}$, PING XIAO $^{5}$, \\ XUE-BIN PENG ${ }^{1}$, SHENG-XIANG ZHANG ${ }^{6}$, JUN-FENG LI $^{1}$ and XIAO-RONG MAO ${ }^{1}$
}

\begin{abstract}
${ }^{1}$ Department of Infectious Diseases and ${ }^{2}$ Hepatology of DongGang Branch, The First Hospital of Lanzhou University, Lanzhou, Gansu 730000; ${ }^{3}$ Department of Infectious Diseases, The Central Hospital of Baoji, Baoji, Shanxi 721008;

${ }^{4}$ Department of Laboratory Medicine; ${ }^{5}$ Institute of Infectious Diseases, The First Hospital of Lanzhou University,

Lanzhou, Gansu 730000; ${ }^{6}$ School of Life Science, Lanzhou University, Lanzhou, Gansu 730000, P.R. China
\end{abstract}

Received October 31, 2014; Accepted July 10, 2015

DOI: $10.3892 / \mathrm{mmr} .2015 .4362$

\begin{abstract}
Bone marrow-derived mesenchymal stem cells (BM-MSCs) are considered to be a potential therapy for end-stage liver disease. However, the therapeutic mechanism of BM-MSCs remains unclear. The aim of the current study was to investigate the role of paracrine signaling in BM-MSCs in liver cirrhosis in vitro. Human BM-MSCs and hepatic stellate cells (HSCs) were cultured using a vertical double cell co-culture system. Groups were divided into HSCs alone (control group) and the co-culture system of BM-MSCs with HSCs (experimental group). HSC morphology was observed by inverted phase contrast microscopy. The proliferative capacity of HSCs was measured with the MTT assay and flow cytometry. Hoechst staining was performed to examine the apoptosis of HSCs. Transforming growth factor (TGF)- $\beta 1$ and Smad7 mRNA expression were detected by reverse transcription-quantitative polymerase chain reaction and western blotting. BM-MSCs did not inhibit the proliferation of HSCs at $24 \mathrm{~h}$, however significantly inhibited the proliferation of HSCs at 48 and $72 \mathrm{~h}$. BM-MSCs additionally induced the apoptosis of HSCs at $48 \mathrm{~h}$. The concentration of TGF- $\beta 1$ in the supernatant at $24 \mathrm{~h}$ and $48 \mathrm{~h}$ in the co-cultured system was observed to be significantly lower than in the control group $(\mathrm{P}<0.05)$. The level of TGF- $\beta 1$ mRNA in the experimental group at $48 \mathrm{~h}$
\end{abstract}

Correspondence to: Ms. Li-Ting Zhang or Ms. Xiao-Rong Mao, Department of Infectious Diseases, The First Hospital of Lanzhou University, 1 West Donggang Road, Lanzhou, Gansu 730000, P.R. China

E-mail: lcheneye@163.com

E-mail:mxr2013@126.com

${ }^{*}$ Contributed equally

Key words: bone marrow-derived mesenchymal stem cells, hepatic stellate cells, transforming growth factor $\beta 1$, Smad7, proliferation, apoptosis was significantly lower than the control group, however Smad7 mRNA levels were significantly greater than in the control group. Additionally, TGF- $\beta 1$ protein levels were significantly lower than in the control group, however levels of Smad7 were greater than the control group. It was concluded that BM-MSCs are able to inhibit the proliferation and promote the apoptosis of HSCs. In addition, the mechanism may be associated with inhibition of the TGF- $\beta 1 /$ Smad pathway in HSCs.

\section{Introduction}

End-stage liver disease is associated with clear patient mortality (1), and orthotopic liver transplantation is the last resort for its treatment. However, due to factors including the shortage of donors, immune rejection and a high cost, the clinical use of orthotopic liver transplantation is restricted (2). Therefore, alternative treatment strategies are urgently required. The activation of hepatic stellate cells (HSCs) and subsequent secretion of large amounts of collagen is considered to be the core mechanism of liver fibrosis $(3,4)$. Bone marrow-derived mesenchymal stem cells (BM-MSCs) possess a wide range of clinical applications in the field of cell therapy and tissue engineering due to their high plasticity, low immunogenicity, rapid amplification rate in vitro and stable genetic background (5).

Liver fibrosis is an early stage of liver cirrhosis, therefore reversing liver fibrosis holds clear clinical potential. An optimal treatment would prevent the development of liver fibrosis, in addition to inhibiting the formation of scar tissue in the liver, in order to stabilize and improve liver function (6). Previous studies have suggested that BM-MSCs may serve a role in the treatment of liver fibrosis (7-9). A previous study identified that the supernatant of cultured BM-MSCs is able to inhibit the expression of matrix metalloproteinase-2 (MMP-2) and tissue inhibitor of matrix metalloproteinase-1 (TIMP-1) in HSCs (10). These results indirectly confirmed that BM-MSCs are able to influence the proliferation of HSCs via the exocrine mechanism. However, it remains unclear which cytokines are secreted by BM-MSCs and which signal transduction pathways 
are used in HSCs. The current study aimed to further elucidate whether BM-MSCs are able to inhibit the proliferation of HSCs, then further explore alterations in transforming growth factor (TGF)- $\beta 1$ during co-culture of BM-MSCs with HSCs.

\section{Materials and methods}

Materials. The human HSC cell line (LX2) was donated by Professor Scott L. Friedman from Icahn School of Medicine at Mount Sinai (New York City, NY, USA). Low glucose-Dulbecco's modified Eagle's medium (L-DMEM) and fetal bovine serum (FBS) (GE Healthcate Life-Sciences, Logan, UT, USA); MTT reagent cartridge (Sigma-Aldrich, St. Louis, MO, USA); the TGF- $\beta 1$ ELISA reagent cartridge (R\&D Systems, Inc., Minneapolis, MN, USA); rabbit anti-human TGF- $\beta 1$ (1:500 dilution; cat.no. BS1361) and rabbit anti-human $\beta$-actin (1:5,000 dilution; cat. no. AP0733) polyclonal antibodies (Bioworld Technology, Inc., St. Louis Park, MN, USA), monoclonal mouse anti-human Smad7 antibody (cat. no. ab55493; Abcam, Pak Shek Kok, Hong Kong); horseradish peroxidase (HRP)-conjugated goat anti-rabbit IgG polyclonal antibody (1:5,000; cat.no. ab6741; Bioworld Technology, Inc.); RNA extraction reagent RNAiao Plus (Takara Bio, Inc., Otsu, Japan); PrimeScript ${ }^{\circledR}$ RT-PCR kit (Takara Bio, Inc.); enhanced chemiluminescence (ECL) light kit (Thermo Fisher Scientific, Inc., Waltham, MA, USA); polyvinylidene difluoride (PVDF) membrane (Merck Millipore, Darmstadt, Germany); semipermeable Transwell insert film (Corning Incorporated, Corning, NY, USA); Hoechst dye (Sigma-Aldrich); bicinchoninic acid (BCA) protein concentration assay kit (Beyotime Institute of Biotechnology, Shanghai, China); protein lysate (Beyotime Institute of Biotechnology).

Culture of human BM-MSCs and HSCs. Human BM-MSCs and HSCs were cultured in L-DMEM containing 10\% FBS at $37^{\circ} \mathrm{C}$ in a $5 \% \mathrm{CO}_{2}$ incubator. Cells were inoculated with a density of $1 \times 10^{5}$ cells $/ \mathrm{ml}$ in culture flasks with a base area of $25 \mathrm{~cm}^{2}$. When cells were $80-90 \%$ confluent, they were washed with phosphate-buffered saline (PBS), digested with $0.25 \%$ trypsin (Thermo Fisher Scientific, Inc.) and subcultured. The growth and morphological characteristics of BM-MSCs and HSCs (third passage) were examined using an inverted phase contrast microscope (AE21; Motic China Group Co., Ltd., Xiamen, China). The growth curve of the cells cultured for 1-9 days was evaluated by MTT assay.

Cell co-culture. The upper and lower double-cell co-culture system was established as previously described $(11,12)$. Cells were cultured in a 6-well plastic cell plate with $2 \mathrm{ml}$ BM-MSCs $\left(1 \times 10^{5}\right.$ cells $\left./ \mathrm{ml}\right)$ in the upper semi-permeable membrane (Transwell insert) and with $2 \mathrm{ml} \mathrm{HSCs}\left(1 \times 10^{5}\right.$ cells/ml) in the lower membrane layer. Cells were divided into two experimental groups: i) Control group, HSCs were cultured alone (upper, L-DMEM media only); and ii) experimental group, BM-MSCs and HSCs were co-cultured. The morphological alterations were examined using an inverted phase contrast microscope at 24 and $48 \mathrm{~h}$ subsequent to incubation.

MTT assay to detect the HSC proliferation inhibition rate. Following co-culture of the cells for 24,48 and $72 \mathrm{~h}$,
$400 \mu \mathrm{l}$ MTT solution ( $5 \mathrm{~g} / \mathrm{l})$ was added to each well. Cells were incubated for $4 \mathrm{~h}$, and the culture medium was discarded. A total of $2 \mathrm{ml}$ dimethyl sulfoxide (Sigma-Aldrich) was added to each well and the wells were agitated for $10 \mathrm{~min}$ in the dark. Once the crystals had fully dissolved, cells were transferred to 96 -well plates, each well containing $150 \mu \mathrm{l}$ and each group consisting of 10 wells. The absorbance value of each well was measured at $490 \mathrm{~nm}$ in an enzyme-linked immunosorbent monitor (Thermo Fisher Scientific, Inc.) and recorded as the A value. The cell growth inhibition rate was calculated as follows: (1 - A value of experimental group / control group A value) $\mathrm{x} 100 \%$.

Flow cytometry to detect apoptosis. Following co-culture for 24 or $48 \mathrm{~h}$, the culture medium was discarded. Cells were washed with PBS and digested with $0.25 \%$ trypsin (excluding EDTA). Cells were resuspended in PBS and centrifuged for $5 \mathrm{~min}$ at $800 \mathrm{x} \mathrm{g}$. The supernatant was discarded and the cells were divided evenly. A total of $5 \mu \mathrm{l}$ annexin $\mathrm{V}$-fluorescein isothiocyanate (FITC) (Beckman Coulter, Inc., Brea, CA, USA) was added into each tube, which were then vortexed prior to incubation in the dark at room temperature for $15 \mathrm{~min}$. A total of $5 \mu \mathrm{l}$ propidium iodide (PI) dye was added to each tube prior to detection for $5 \mathrm{~min}$. Prior to detection, $200 \mu \mathrm{l}$ $1 \mathrm{X}$ binding buffer was added into the tubes.

Hoechst staining of apoptotic cell bodies. Subsequent to co-culture for 24 and $48 \mathrm{~h}$, the culture medium was removed and $2 \mathrm{ml} \mathrm{4 \%} \mathrm{formaldehyde} \mathrm{was} \mathrm{added} \mathrm{into} \mathrm{each} \mathrm{well.} \mathrm{Cells}$ were fixed for $15 \mathrm{~min}$ at room temperature and were washed twice with PBS, for 5 min each time. HSCs were stained with Hoechst dye for 6-9 min in the dark at room temperature. Apoptotic cell bodies in HSCs were examined using a fluorescence microscope (BX431-46; Olympus Corporation, Tokyo, Japan).

ELISA for TGF- $\beta 1$ levels. Following co-culture for 24 and $48 \mathrm{~h}$, the supernatants from the upper and lower Transwell chambers were collected and centrifuged at $750 \mathrm{x}$ g for $20 \mathrm{~min}$. TGF- $\beta 1$ concentration was measured in accordance with the ELISA kit (R\&D Systems, Inc.) instructions.

Total RNA extraction of HSCs and reverse transcription-quantitative polymerase chain reaction detection. HSCs were collected and counted at $48 \mathrm{~h}$ in each group. The primer sequences used were as follows: $\beta$-actin, upstream sequence TGGCACCCAGCACAATGAA and downstream sequence CTAAGTCATAGTCCGCCTAGAAGCA; TGF- $\beta 1$, upstream sequence TGGAAACCCACAACGAAATC and downstream sequence GTATTTCTGGTACAGCTCCA; Smad7, upstream sequence TCTGCGAACTAGAGTCTCCC and downstream sequence ACGCACCAGTGTGACCGATC. The total RNA was extracted using RNAiso Plus reagent and RNA integrity and concentration were measured in the spectrophotometer (Tecan Group Ltd., Männedorf, Switzerland). A total of 500 ng RNA was used to synthesize $10 \mu \mathrm{l}$ cDNA using the PrimeScript ${ }^{\circledR}$ RT-PCR kit. The reaction system volume was $10 \mu \mathrm{l}$. The reaction conditions were as follows: $37^{\circ} \mathrm{C}$ for $15 \mathrm{~min}$ then $85^{\circ} \mathrm{C}$ for $5 \mathrm{sec}$. Genes were amplified using a LightCycler amplification system (Bio-Rad Laboratories, Inc., Hercules, 
A

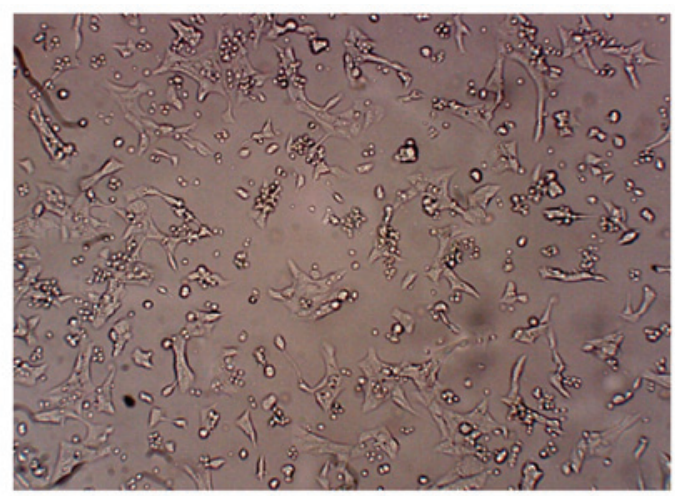

B

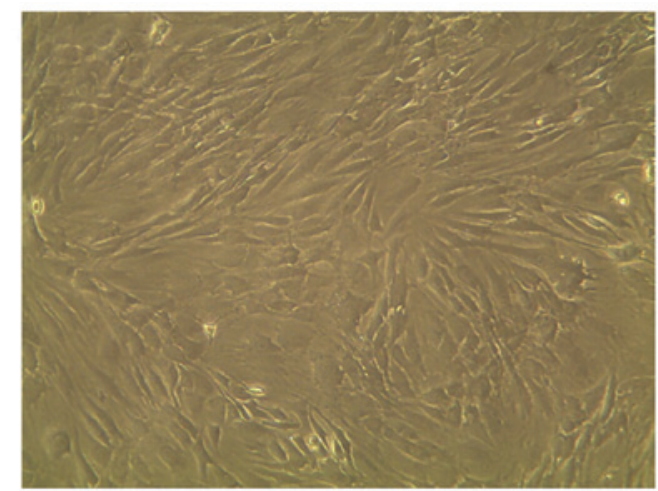

C

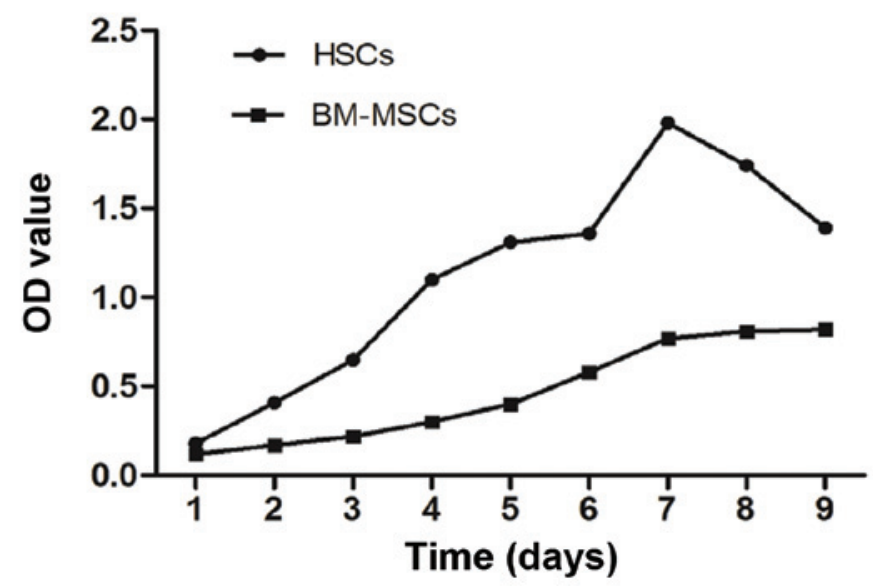

Figure 1. The in vitro growth of human BM-MSCs and HSCs. (A) The morphology of HSCs subsequent to being passaged for 72 h (the 3rd generation; magnification, x100); (B) the morphology of human HSCs subsequent to being passaged for 24 h (the 3rd generation; magnification, x100); (C) the growth curve of HSCs and BM-MSCs. BM-MSCs, bone marrow-derived mesenchymal stem cells; HSCs, hepatic stellate cells; OD, optical density.

CA, USA). The reaction system volume was $20 \mu 1$. The reaction conditions were as follows: $95^{\circ} \mathrm{C}$ for $30 \mathrm{sec}\left(20^{\circ} \mathrm{C} / \mathrm{sec}\right)$ for 1 cycle, then at $95^{\circ} \mathrm{C}$ for $5 \mathrm{sec}\left(20^{\circ} \mathrm{C} / \mathrm{sec}\right)$ and $60^{\circ} \mathrm{C}$ for $20 \mathrm{sec}$ $\left(20^{\circ} \mathrm{C} / \mathrm{sec}\right)$ for 40 cycles. TGF- $\beta 1, \mathrm{Smad} 7 \mathrm{mRNA}$ expression levels were detected. The data were stated as $2^{-\Delta \Delta \mathrm{Ct}}$.

HSCs total protein extraction and western blot analysis. Following co-culture for 24 and 48 h, cells were lysed and total protein extracted from HSCs. Protein content was measured using the BCA protein concentration determination kit. The sample quantity was $30 \mu \mathrm{g}$. Proteins were separated by SDS-PAGE (10\% gel; Bio-Rad Laboratories, Inc.), transferred to a PVDF membrane, blocked with Tris-buffered saline and Tween 20 and 5\% skimmed milk and incubated with anti-human TGF- $\beta 1(1: 500)$ at $4{ }^{\circ} \mathrm{C}$ overnight. The membrane was incubated with the HRP-conjugated secondary antibody, then developed using ECL. Data were analyzed using Image J 3.0 (Bio-Rad Laboratories, Inc.). The gray ratio of TGF- $\beta 1$ proteins and $\beta$-actin represented the relative level of the target protein. Smad7 protein was detected as mentioned above.

Statistical analysis. SPSS software, version 18.0 (SPSS, Inc., Chicago, IL, USA) was used for statistical analysis. The count data were analyzed using the Chi-square test, measurement data were subjected to Student's t-test. Experimental data are expressed as mean \pm standard deviation. $\mathrm{P}<0.05$ was considered to indicate a statistically significant difference.

\section{Results}

Culture of BM-MSCs and HSCs. HSCs were observed to be adherent and oval or spindle-shaped, and cytoplasmic lipid droplets appeared following inoculation for 2-3 h, with certain cells beginning to stretch out during this time. Subsequent to culture of HSCs for 2-3 days, cells were 80-90\% confluent and could be sub-cultured. Lipid droplets in the cytoplasm gradually reduced or disappeared, and cells displayed pseudopodia. HSCs exhibiting rapid growth and proliferation when passaged for 3-4 generations were used for the experiments (Fig. 1A). Cultured BM-MSCs were observed to be round or oval and were of varying sizes following inoculation. Subsequent to the media being replaced, cell adherence increased and the cells became uniform in shape, tightly packed, multi-fusiform and began to merge into a sheet. When cells were digested by trypsin, they became round, began to adhere, gradually restoring their long spindle morphology over 2-3 h. Subsequent to culture for $72 \mathrm{~h}, \mathrm{BM}-\mathrm{MSC}$ were highly confluent, of uniform shape and fused with typical swirling growth (Fig. 1B). The third passage cells were for transplantation. The growth curve of HSCs and BM-MSC demonstrated that HSCs grew faster than BM-MSCs (Fig. 1C).

Inhibitory effect of BM-MSC on HSCs. Hoechst staining revealed the number of apoptotic bodies in the experimental group was significantly increased compared with the control group (Fig. 2A-D). The inhibitory effect of BM-MSCs on HSCs 

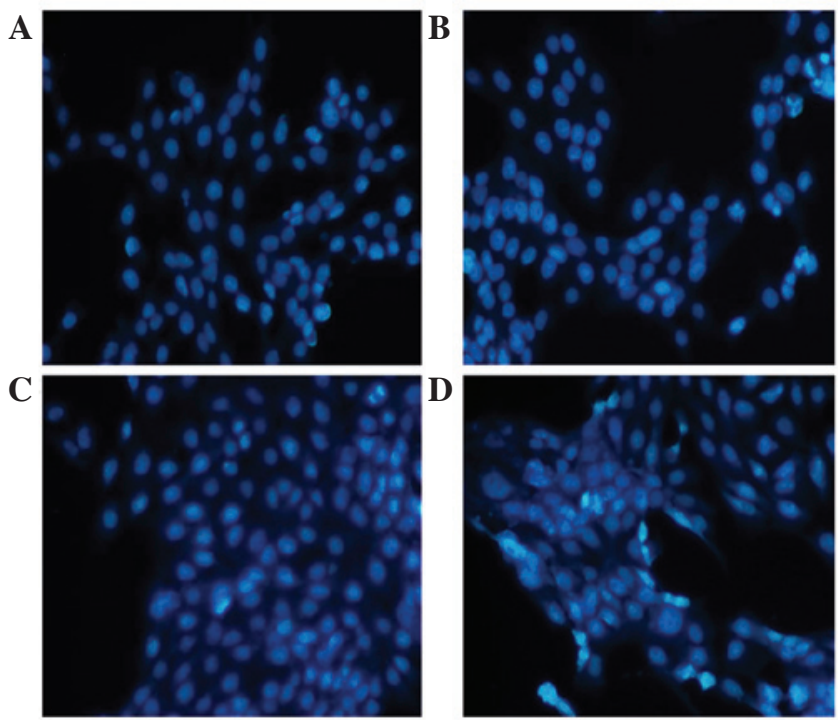

$\mathbf{E}$

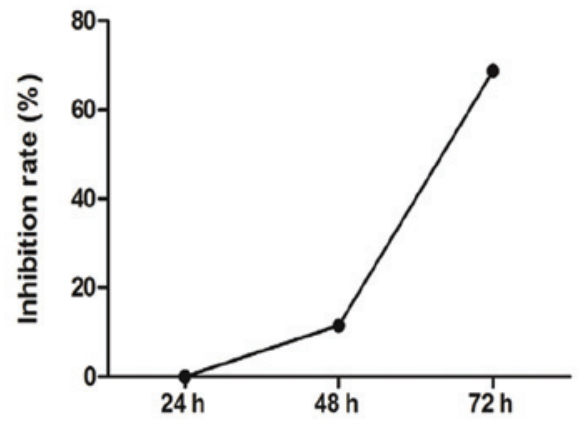

Figure 2. Apoptosis and inhibition of proliferation of HSCs caused by BM-MSCs. (A) Control group at $24 \mathrm{~h}$; (B) experimental group at $24 \mathrm{~h}$; (C) control group at $48 \mathrm{~h}$; (D) experimental group at $48 \mathrm{~h}$ ); (E) the proliferation rate of HSCs by BM-MSCs. BM-MSCs, bone marrow-derived mesenchymal stem cells; HSCs, hepatic stellate cells.

was detected by MTT assay following culture of the cells for 24,48 and $72 \mathrm{~h}$. The absorbance values of the control group at 24,48 and $72 \mathrm{~h}$ (at $490 \mathrm{~nm}$ ) were $0.149 \pm 0.012,0.405 \pm 0.007$ and $3.146 \pm 0.033$, respectively. The absorbance values of the experimental group were $0.149 \pm 0.160,0.358 \pm 0.007$ and $0.986 \pm 0.020$, respectively. There was no significant difference between the control and experimental group at $24 \mathrm{~h}(\mathrm{P}=1.000)$, however the absorbance values at 48 and $72 \mathrm{~h}$ between the two groups were significantly different $(\mathrm{P}<0.001)$. BM-MSCs inhibited the proliferation of HSCs, with the inhibitory rates of BM-MSCs on HSCs at 24, 48 and $72 \mathrm{~h}$ observed to be $0,11.5$ and $68.7 \%$, respectively. BM-MSCs did not inhibit the proliferation of HSCs at $24 \mathrm{~h}$, however significantly inhibited the proliferation of HSCs in a time-dependent manner at 48 and $72 \mathrm{~h}$ (Fig. 2E).

Apoptotic effect of BM-MSCs on HSCs. Following co-culture of BM-MSCs with HSCs for 24 and $48 \mathrm{~h}$, cells were double stained with annexin V-FITC and PI to detect the rate of apoptosis of HSCs using flow cytometry (Fig. 3A-D). The rate of apoptosis was not identified to significantly differ between the control and experimental groups at $24 \mathrm{~h}(2.08 \%$ for control group and $1.00 \%$ for experimental group). However, the rate of apoptosis was significantly increased in the experimental group at $48 \mathrm{~h}$ ( $7.51 \%$ for control group and $10.28 \%$ for experimental group) (Fig. 3E).
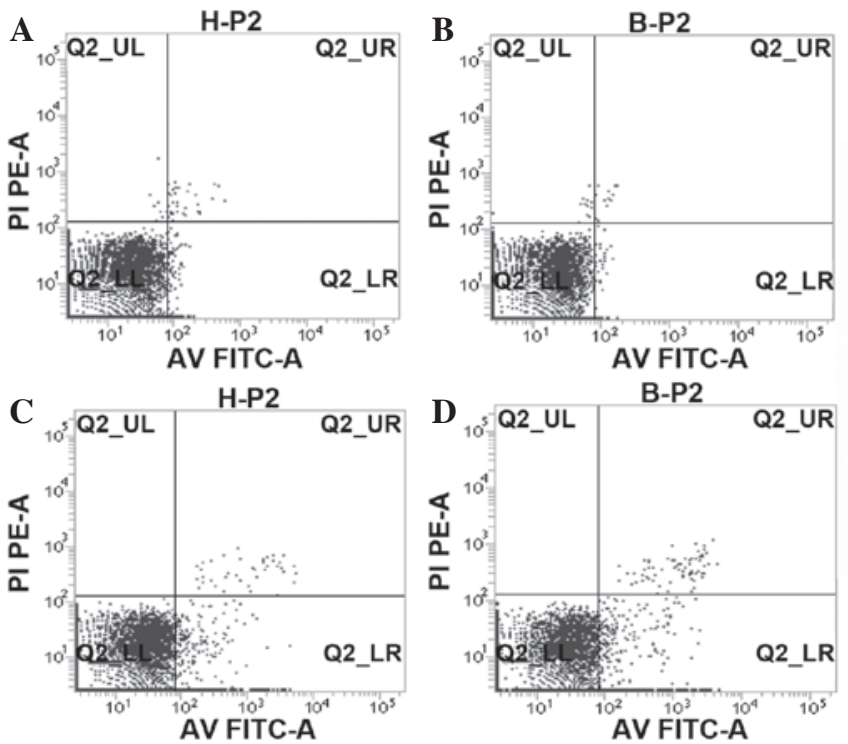

$\mathbf{E}$

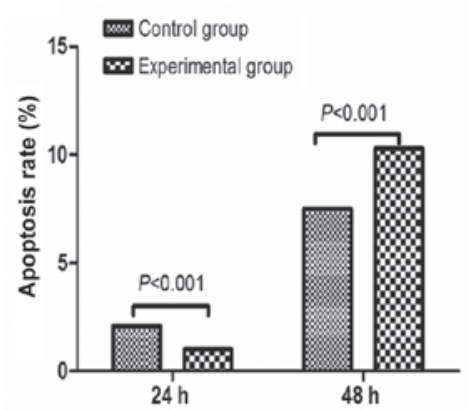

Figure 3. The apoptotic rate of HSCs caused by BM-MSCs. (A) Apoptosis rate of control group at $24 \mathrm{~h}$; (B) apoptotic rate of experimental group at $24 \mathrm{~h}$; (C) apoptotic rate of control group at $48 \mathrm{~h}$; (D) apoptotic rate of experimental group at $48 \mathrm{~h}$; (E) the apoptotic effect of BM-MSCs on HSCs at 24 and $48 \mathrm{~h}$. BM-MSCs, bone marrow-derived mesenchymal stem cells; HSCs, hepatic stellate cells; PI, propidium iodide; AV FITC; annexin V fluorescein isothiocyanate.

Expression of TGF- $\beta 1$ and SMAD7 mRNA. The TGF- $\beta 1$ and Smad7 mRNA expression levels in the control and experimental groups were as follows: Control group, $1.00 \pm 0.00$ and $1.00 \pm 0.00$, respectively; experimental group, $0.401 \pm 0.301$ and $9.697 \pm 2.591$, respectively. Following co-culture for $48 \mathrm{~h}$, TGF- $\beta 1$ mRNA in the experimental group was significantly lower than in the control group, and Smad7 mRNA was significantly higher than that in the control group $(\mathrm{P}<0.05$; Fig. 4).

Concentration of TGF- $\beta 1$ in the supernatant. To clarify whether BM-MSCs impact the secretion of TGF- $\beta 1$ from HSCs, the TGF- $\beta 1$ concentration in the supernatant was measured in the BM-MSCs and HSCs co-culture group, BM-MSCs group and the HSCs group at 24 and $48 \mathrm{~h}$. The concentration of TGF- $\beta 1$ in the BM-MSCs single culture group was below the ELISA kits minimum detection value, thus could not be measured. The concentration of TGF- $\beta 1$ in the co-culture group was significantly lower than in the HSC single culture group at 24 and $48 \mathrm{~h}(\mathrm{P}<0.05$; Fig. 5).

Expression of TGF- $\beta 1$ and Smad7 protein. The expression of TGF- $\beta 1$ and Smad7 protein was measured in the control group and experimental group at 24 and $48 \mathrm{~h}$, and levels were observed 


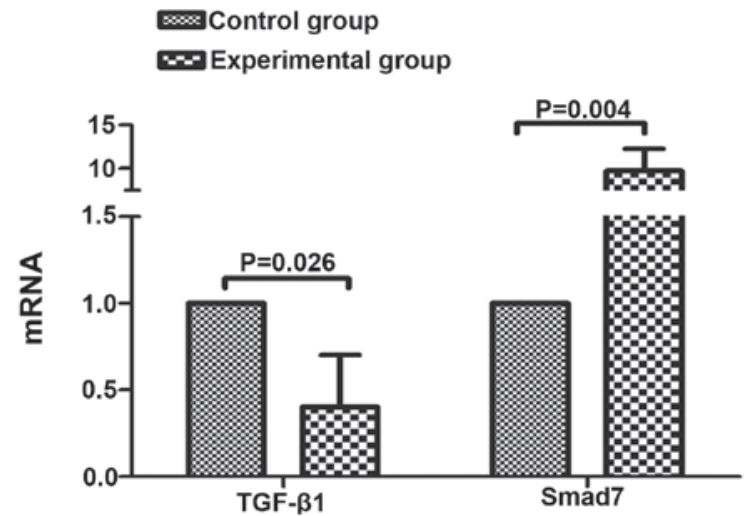

Figure 4 . The mRNA expression of TGF- $\beta 1$ and Smad7. TGF- $\beta 1$, transforming growth factor $\beta 1$.

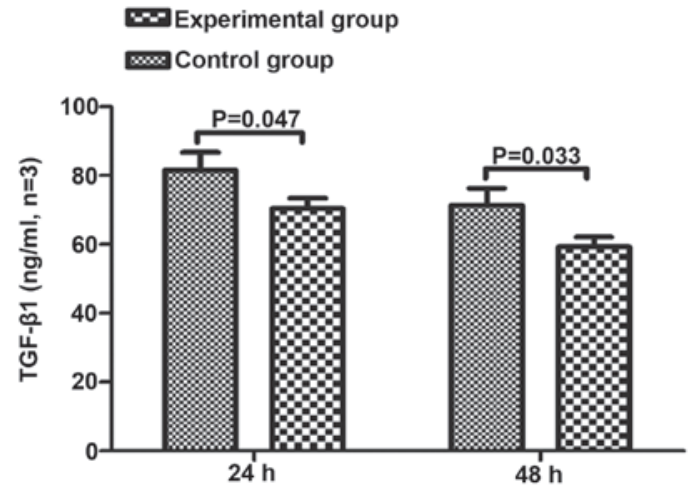

Figure 5. Impact of BM-MSCs on secretion of TGF- $\beta 1$ from HSCs. BM-MSCs, bone marrow-derived mesenchymal stem cells; TGF- $\beta 1$, transforming growth factor $\beta 1$; HSCs, hepatic stellate cells.

A

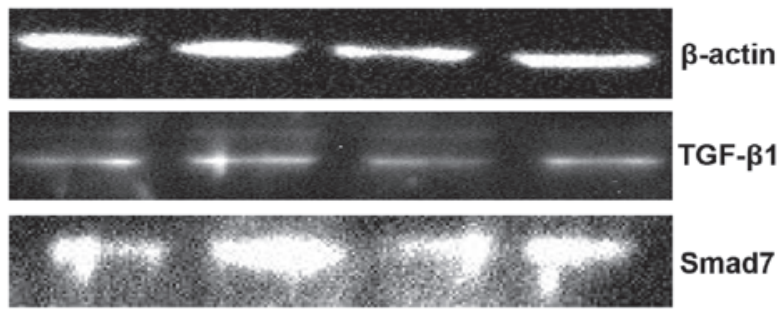

B

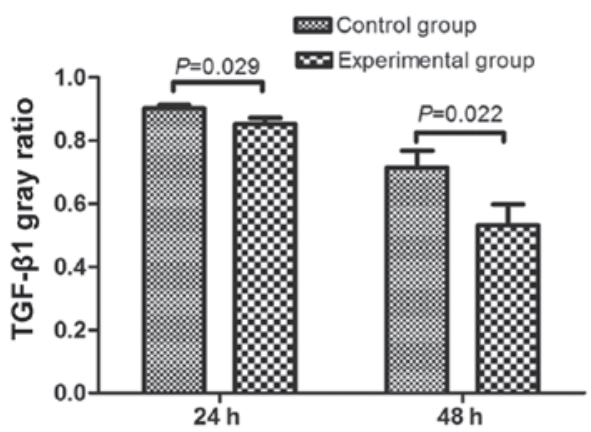

C

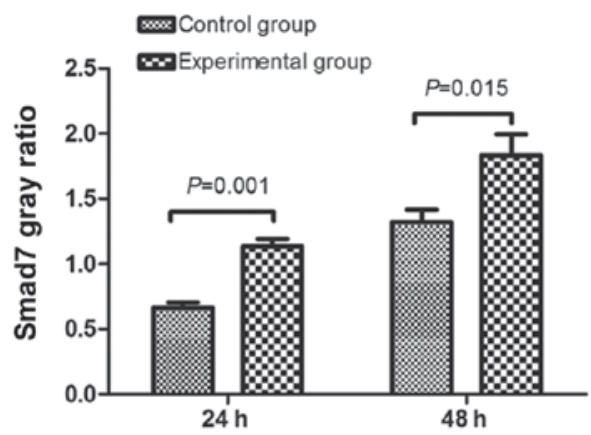

Figure 6. The expression of TGF- $\beta 1$ and Smad7 protein in the control and experimental groups. (A) TGF- $\beta 1$ and Smad7 expression. From left to right: Control group at $24 \mathrm{~h}$, experimental group at $24 \mathrm{~h}$, control group at $48 \mathrm{~h}$ and the experimental group at $48 \mathrm{~h}$. Quantification of the protein expression levels of (B) TGF- $\beta 1$ and (C) Smad7. TGF- $\beta 1$, transforming growth factor $\beta 1$.

to be significantly different between the groups (Fig. 6). Expression of TGF- $\beta 1$ protein in the experimental group was lower than in the control group at 24 and $48 \mathrm{~h}(24 \mathrm{~h}$ control group, $0.902 \pm 0.012 ; 24$ h experimental group, $0.854 \pm 0.018$; $48 \mathrm{~h}$ control group, $0.715 \pm 0.053 ; 48 \mathrm{~h}$ experimental group, $0.532 \pm 0.066$ ), and Smad7 protein levels were higher than that of the control group ( $24 \mathrm{~h}$ control group, $0.661 \pm 0.043$; $24 \mathrm{~h}$ experimental group, $1.134 \pm 0.059 ; 48 \mathrm{~h}$ control group, $1.322 \pm 0.095 ; 48 \mathrm{~h}$ experimental group, $1.834 \pm 0.161$ ).

\section{Discussion}

Liver fibrosis is an early stage in the development of liver cirrhosis. In-depth study of the mechanisms controlling the development of liver fibrosis may aid in reducing the occurrence of liver fibrosis (13), thereby preventing the progression of liver cirrhosis and improving quality of life for patients.
Previous studies have confirmed that HSCs serve a key role in the development of liver fibrosis $(14,15)$. Following the activation of HSCs by a variety of stimulating factors, they are able to proliferate, secrete excessive TIMP-1 and inhibit MMP-1 activity, leading to an imbalance between collagen deposition and degradation (16). TGF- $\beta 1$ is a key factor for the promotion of HSCs activation and expression of the extracellular matrix (17).

In the present study, a co-culture system was established in which BM-MSCs cannot pass through the Transwell insert while cytokines are able to pass freely. BM-MSCs were seeded in the upper layer and HSCs in the lower layer, and the proliferative and apoptotic effects of BM-MSCs on HSCs were observed. The results of the MTT assay suggested that BM-MSCs did not significantly inhibit the proliferation of HSCs at $24 \mathrm{~h}$. However, BM-MSCs significantly inhibited the proliferation of HSCs when they were co-cultured for 
48 and 72 h. Additionally, BM-MSCs did not promote apoptosis of HSCs at $24 \mathrm{~h}$, however induced significant apoptosis at $48 \mathrm{~h}$. The number of apoptotic cell bodies in the experimental group was significantly increased compared with the control group. Therefore, it was concluded that BM-MSCs inhibit proliferation and induce apoptosis in HSCs. The non-contact co-culture method excluded the contact inhibition effect of BM-MSC on HSCs. These data suggest that BM-MSCs may secrete cytokines that pass through the Transwell insert mesh and inhibit proliferation and promote apoptosis of HSCs. The present study demonstrated that HSCs synthesise and secrete a reduced quantity of TGF- $\beta 1$ and greater Smad7 following co-culture with BM-MSCs. These data further confirm that BM-MSCs inhibit the proliferation and induce apoptosis of HSCs through the secretion of certain cytokines involved in the TGF $\beta /$ Smad signaling pathway. Further experiments are required to identify the cytokines secreted by BM-MSCs.

In conclusion, the current study indicated that BM-MSC may serve a role in inhibiting the proliferation of HSCs and promoting apoptosis of HSCs through paracrine signaling, suggesting that infusion of the BM-MSC culture supernatant may be used to clinically treat liver fibrosis patients. However, in the present study TGF- $\beta 1$ and Smad7 mRNA and protein expression levels were observed to be altered following co-culture for $24 \mathrm{~h}$. However, from the results at $24 \mathrm{~h}$, the alterations of TGF- $\beta 1$ and Smad7 did not appear to affect cell proliferation and apoptosis during this time. This suggests that the effect of alterations in fibrogenic factors and fibrosis inhibitors on the mRNA and protein levels in HSCs co-cultured for $24 \mathrm{~h}$ may not be large enough to inhibit proliferation and promote apoptosis of HSCs. Following $48 \mathrm{~h}$ of co-culture, alterations at the gene and protein levels were observed, in addition to inhibition of HSC proliferation, and an increase in the number of apoptotic cells. In the early stages of the experiment, three observation time points were used, 24, 48 and $72 \mathrm{~h}$, however from the MTT assay it was established that co-culture of BM-MSCs and HSCs for $72 \mathrm{~h}$ significantly inhibited the proliferation of HSCs. Additionally, the density of HSCs in the control group culture was observed to impact upon levels of apoptosis, with the number of adherent cells unable to meet the requirements of the follow-up experiments. Therefore, two observation time points, 24 and $48 \mathrm{~h}$, were used in the follow-up experiment.

There are several limitations of the current study. The experiments used cultured BM-MSCs and HSCs cell lines in vitro, which cannot accurately reflect the effect of BM-MSCs in vivo. In addition, the results did not account for the influence of the inoculation on cell concentration. Therefore, future studies should address these limitations.
In summary, the present study identified that BM-MSCs are able to inhibit the proliferation of HSCs and promote their apoptosis, and the mechanism may be associated with inhibition of the TGF- $\beta 1 /$ Smad pathway in HSCs.

\section{References}

1. Mutimer DJ and Lok A: Management of HBV- and HCV-induced end stage liver disease. Gut 61 (Suppl 1): i59-i67, 2012.

2. Olson JC, Wendon JA, Kramer DJ, Arroyo V, Jalan R, Garcia-Tsao G and Kamath PS: Intensive care of the patient with cirrhosis. Hepatology 54: 1864-1872, 2011.

3. Friedman SL: Hepatic stellate cells: Protean, multifunctional, and enigmatic cells of the liver. Physiol Rev 88: 125-172, 2008.

4. Kisseleva $T$ and Brenner DA: Role of hepatic stellate cells in fibrogenesis and the reversal of fibrosis. J Gastroenterol Hepatol 22 (Suppl 1): S73-S78, 2007.

5. Sancho-Bru P, Najimi M, Caruso M, Pauwelyn K, Cantz T, Forbes S, Roskams T, Ott M, Gehling U, Sokal E, et al: Stem and progenitor cells for liver repopulation: Can we standardise the process from bench to bedside? Gut 58: 594-603, 2009.

6. Mormone E, George J and Nieto N: Molecular pathogenesis of hepatic fibrosis and current therapeutic approaches. Chem Biol Interact 193: 225-231, 2011.

7. Rabani V, Shahsavani M, Gharavi M, Piryaei A, Azhdari Z and Baharvand $\mathrm{H}$ : Mesenchymal stem cell infusion therapy in a carbon tetrachloride-induced liver fibrosis model affects matrix metalloproteinase expression. Cell Biol Int 34: 601-605, 2010.

8. Mohamadnejad M, Alimoghaddam K, Bagheri M, Ashrafi M, Abdollahzadeh L, Akhlaghpoor S, Bashtar M, Ghavamzadeh A and Malekzadeh R: Randomized placebo-controlled trial of mesenchymal stem cell transplantation in decompensated cirrhosis. Liver Int 33: 1490-1496, 2013.

9. Nunes de Carvalho S, Helal-Neto E, de Andrade DC, Costa Cortez EA, Thole AA, Barja-Fidalgo C and de Carvalho L: Bone marrow mononuclear cell transplantation increases metalloproteinase- 9 and 13 and decreases tissue inhibitors of metalloproteinase- 1 and 2 expression in the liver of cholestatic rats. Cells Tissues Organs 198: 139-148, 2013.

10. Wang S, Zhang LT and Chen H: Effect of human bone marrow mesenchymal stem cells culture supernatant on related enzyme expression in hepatic stellate cells. J Clin Rehab Tissue Eng Res 16: 8374-8379, 2012.

11. Parekkadan B, van Poll D, Megeed Z, Kobayashi N, Tilles AW, Berthiaume $F$ and Yarmush ML: Immunomodulation of activated hepatic stellate cells by mesenchymal stem cells. Biochem Biophys Res Commun 363: 247-252, 2007.

12. Shi L, Li G, Wang J, Sun B, Yang L, Wang G, Wang D, Mu L, Chen H, Jin L, et al: Bone marrow stromal cells control the growth of hepatic stellate cells in vitro. Dig Dis Sci 53: 2969-2974, 2008.

13. Friedman SL: Hepatic fibrosis - overview. Toxicology 254: 120-129, 2008.

14. Friedman SL: Mechanisms of disease: Mechanisms of hepatic fibrosis and therapeutic implications. Nat Clin Pract Gastroenterol Hepatol 1: 98-105, 2004.

15. Friedman SL: Seminars in medicine of the Beth Israel Hospital, Boston. The cellular basis of hepatic fibrosis. Mechanisms and treatment strategies. N Engl J Med 328: 1828-1835, 1993.

16. Friedman SL: Mechanisms of hepatic fibrogenesis. Gastroenterology 134: 1655-1669, 2008.

17. Li JH, Huang XR, Zhu HJ, Johnson R and Lan HY: Role of TGF-beta signaling in extracellular matrix production under high glucose conditions. Kidney Int 63: 2010-2019, 2003. 